\title{
Restructuration of distribution power system using Genetics Algorithms
}

\author{
K. Sebaa, N. Henini, A. Tlemçani \\ Laboratoire de Génie Electrique \\ Université Mouloud Mammeri de Tizi-Ouzou \\ sebaa.karim@univ-medea.dz
}

\author{
H. Nouri \\ Power Systems, Electronics and Control Research Group \\ Department of Engineering Design and Mathematics, \\ University of West of England, Bristol, BS16 1QY, U.K. \\ Hassan.Nouri@uwe.ac.uk
}

\begin{abstract}
This paper uses Genetic Algorithms (GAs) to solve the optimal reconfiguration in radial distribution systems for power loss reduction that determine the optimal switches. The GA is a relatively powerful intelligence evolution method for solving optimization problems. It is a population based approach that was inspired from natural behavior of species. In this paper $\mathbf{G A}$ is applied to a realistic distribution system (106 buses) located in the medea city (Algeria). For the comparison purpose, our method is compared with the classical Branch and Bound (B\&B) method, widely used by the Distribution companies. The results confirm the superiority of the GA.
\end{abstract}

Index Terms-- GA; B\&B; losses; loading and reconfiguration.

\section{INTRODUCTION}

In the Distribution Power System (DPS); to reduce the losses and improving the voltage profile the reconfiguration of DPS is an alternative way which not require any investment. This operation must be considered when planning the operation due to the high variable costs associated with these systems [1].

The problem of reconfiguration involves of the definition of the states (open or closed) of the maneuverable switches attached to certain sections of the distribution network [2]. In the MV PDS these maneuver devices include (i) sectionalizing or normally closed (NC) switches and (ii) tie or normally open (NO) switches. In LV DPS its are a simple conductors to be connected or disconnected. This option is used to determine a connected and radial network topology that minimizes losses and voltage deviations [3].

As this problem includes combinatorial variable (0 and 1) with nonlinear objective function and constrained, models of integer nonlinear programming (INLP) are used. These models must consider the integer nature of the problem because the number of possible solutions grows exponentially with the number of discrete variables [4]. Also, the radial and connected topographies of the DPS present additional complexity for the solution techniques.

The heuristic-based methods have been proposed [5-11] in order to reduce the search space associated with reconfiguration problem.
The use of the meta-heuristics for INLP problems give a well exploration of the search space. These algorithms permit the transition between local optima of the feasible region, as well as a more focused search in each subspace. Algorithms based on meta-heuristics, such as Genetic Algorithms [12-16], Simulated Annealing [17,18], Artificial Ant Colony [19] and Tabu Search [20,21], have been used to solve the problem of EDS reconfiguration. With the same purpose in mind, Ref. [22] presents an algorithm based on Artificial Immune Systems to reduce active power losses. In [23], a method based on the bacterial foraging optimization algorithm is proposed for DPS reconfiguration and loss minimization. Some modifications have been developed to retain the radial structure and hg|reduce the searching requirement. The work in [24] presents a method for the reconfiguration and phase balancing of DPS based on a bacterial foraging approach using a fuzzy multi-objective function.

Other family called Determinist techniques can be used to deal with this problem; these techniques are called Branch and Bound which are the extension of the simplex method to INLP.

In this paper, the comparison between $\mathrm{GA}$ and $\mathrm{B} \& \mathrm{~B}$ algorithm for the problem of DPS reconfiguration will be discussed, Also, an application to a realistic DPS located in Medea, Algeria is presented.

\section{PROBLEM STATEMENT}

For a given distribution power system, the problems that are proposed to solve are to determine optimal operating schemes according to specified criteria and constraints.

\section{A. Distribution Power System Structure}

The structure of a network is defined by the constructive and invariant arrangement of all of its components: stations lines, cables etc...

In graphic terms, this structure is determined by:

- A finite set $X=\left\{x_{1}, x_{2}, x_{3}, \mathrm{~K}, x_{N}\right\}$ of elements called vertices or buses. Where $N$ is the total number of buses. 
- A finite set $B=\left\{b_{1}, b_{2}, b_{3}, \mathrm{~K}, b_{M}\right\}$ of elements called links or branches, where $M$ is the total number of branches. Each branch allows to connecting the two components of a pair belonging to a subset of dimension $\mathrm{M}$ and included in the Cartesian product:

$$
X^{\prime} X=\left\{\left(x_{i}, x_{j}\right) / x_{i} \hat{\mathrm{I}} X \text { and } x_{j} \hat{\mathrm{I}} X\right\}
$$

The sets $X$ and $B$ correspond to the axiomatic definition of a graph, denoted $G(X, B)$ [Ber83], the orientation of each branch can be a priori any or indefinite (Fig. 1)

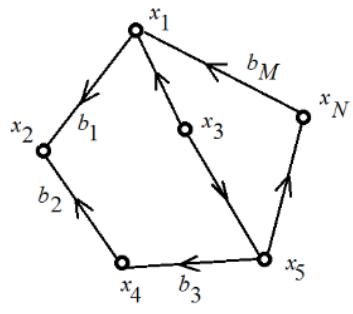

$G(X, B)$

Fig. 1. Structure of a Distribution Power System

Network topology, synonym of its operating diagram corresponds to the permanent or temporary assembly of its components.

In graphic terms, this topology is associated with a sub-graph of $G$, defined by the set $X$ and a set of links $L$ such that $L$ İ $B$.

The links belonging to $L$ mean that each of them can exchange a flow (current) between the two corresponding nodes. It said, in this case, that the link is in service. For each links in the complementary set, this exchange is interrupt intentionally and it said that the link is out of service.

In the case of distribution power systems, the partial graph is connected, i.e., it is possible to achieve, from a given node any other node via a path composed by the elements of $L$. In addition, the number of branches belonging to $L$ is the total number bus minus 1 .

This particular partial graph of $\mathrm{G}$ is called a tree, which will be denoted $T(X, L)$.

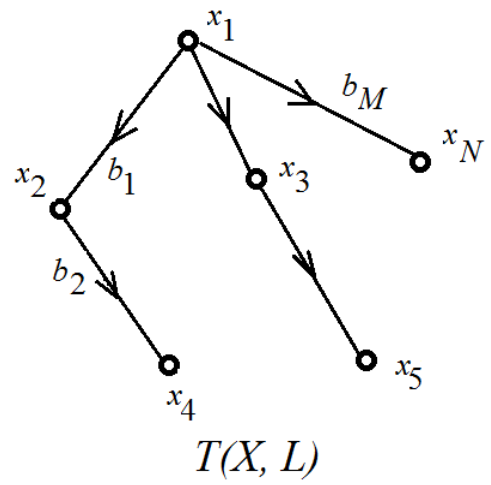

Fig. 2. Topology of a Distribution Power System

\section{B. General mathematic formulation}

The problem to solve is to determine the optimal exploitation schemes in accordance with specified criteria and constraints. In this section, it is proposed to express it mathematically.

For a distribution network defined by its structure, or by the its graph, it is associated to the set $B$ a set $Y$ and a set $U$ such that: $Y \quad$ set of state variables representing the currents in the branches

$U \quad$ set of decision variables representing the topologic stats of branches; such as:

" $u_{k}$ I $U \begin{array}{ll}u_{k}=1 & \text { if the branch } b_{k} \text { is in service } \\ u_{k}=0 & \text { if the branch } b_{k} \text { is out of service }\end{array}$

Also, it is associated with the set $X$ a set $Z$ such as:

$Z \quad$ set of state variables representing the voltage at the nodes.

According to this notation, an optimization problem can be formuled. Where the components of $Y, Z$ and $U$ should be defined (1).

$$
\begin{aligned}
& \min f(Y, Z, U) \text { objective function } \\
& \text { s.t. } \\
& g(Y, Z, U)=0 \text { Kirchoff's law } \\
& h(Y, Z, U) £ 0 \text { security constraints } \\
& s(U)=1 \quad \text { radiality } \\
& t(U) £ 0 \quad \text { number of switch }
\end{aligned}
$$

\section{Kirchhoff equations}

This constraint laid by the Lemma of Kirchhoff about the currents.

$$
\stackrel{\circ}{a=1}_{k}^{M} u_{k} \times a_{i k} \times I_{k}=J_{i}
$$

where

$$
a_{i k}=\begin{array}{ll}
\frac{1}{1}+1 & \text { if } b_{k} \text { is oriented to } x_{i} \\
\hline & \text { if } b_{k} \text { is oriented from } x_{i} \\
0 & \text { if } x_{i} \text { is not an end of } b_{k}
\end{array}
$$

$I_{k} \quad$ is the current in the branch $b_{k}$

$J_{i} \quad$ is the load current at the bus $x_{i}$

This constraint also involves the continuity of service, ie the desire to fully meet the load at any node of the network.

Since the solution of the optimization problem must ansure that the network is radial without isolating

any load buses, the second lemma of Kirchhoff about the tensions will be implicitly respected.

\section{Radiality}

This constraint is related to decision variables. It involves the conservation of the radially operation of the network.

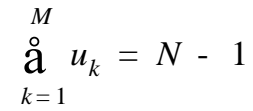


To complete the constraint [3]; It is necessary to impose the connectivity of the operating diagram. It can be expressed as follows:

$$
" x_{i}, x_{j} \$ L_{i} \text { È } L_{j} \text { whether } \underset{b_{i} \hat{\mathrm{I}} L_{i}}{\tilde{\mathrm{O}}} u_{i} \times \underset{b_{j} \hat{\mathrm{I}} L_{j}}{\tilde{\mathrm{O}}} u_{j}=1
$$

where $L_{i}$ È $L_{j}$ is a path connecting the buses $x_{i}$ and $x_{j}$ and where $u_{i}$ and $u_{j}$ denote the topological states of the branches of $L_{i}$ and $L_{j}$ constituting this path.

\section{E. inequality constraints}

1) Security: change the default, adjust the template as follows. These constraints imply only the state variables, namely the branches currents and the buses voltages, must not exceed the allowable limits.

For current, the security constraint is expressed for each branch by the following inequality:

$$
\left|I_{k}\right| £ u_{k} \times I_{k}^{\max }
$$

where

$I_{k}^{\max } \quad$ is the thermal limit of current in the branch $b_{k}$

$I_{k} \quad$ is the current in the branch $b_{k}$

Operators must ensure voltage as close as possible to the nominal voltage at each consumption point; the maximum tolerated deviation can vary from one company to another. Generally, the absolute value of this difference varies between 5 and $10 \%$ depending on the normal operation or failure mode of the network. The expression of this constraint is:

$$
\left|V_{\text {in }}-V_{i}\right| / V_{\text {in }} \times 100 £ 5 \%
$$

$V_{i n}, V_{i}$ are respectively the nominal voltage and the voltage at the bus $x_{i}$

2) Number of switching operations: Due to failure of a branch, operators wish to restore the continuity of service using a backup operating scheme that is as close as possible to the original topology. This desire led to the limitation of the number of switching operations:

$$
\stackrel{\circ}{\circ}_{k=1}^{\circ}\left|u_{k}-u_{k}^{0}\right| £ \mathrm{D}_{\max }
$$

$u_{k}^{0} \quad$ is the initial topology of the branch $b_{k}$

$\mathrm{D}_{\max }$ is the maximum number of allowed switching

\section{F. Objective function}

1) Losses: It is inevitable and cause problems for both technical and economic. They can expressed according to the following expression

$$
3 \times \stackrel{a}{0}_{k=1}^{M} R_{k} \times I_{k}^{2}
$$

$R_{k}$ is the resistance of the branch $b_{k}$
$I_{k}$ is the current of the branch $b_{k}$

2) Load balancing: The load balancing results from the desire to exploit the network optimally by dividing current reserves uniformly.

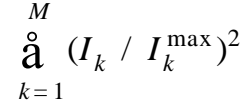

3) Maximum voltage deviation: the aim of this objective is to minimize the maximum voltage deviation

$$
\max \left|V_{\text {in }}-V_{i}\right| / V_{\text {in }} i=1, \mathrm{~K}, N
$$

\section{GENETICS ALGORITHMS}

Here, we descript the use of the GA to solve the problem of the reconfiguration of a DPS. The GA used in this work is the original algorithm presented by David E. Goldberg in [25].

1) Fitness:

As the GA is an unconstrained optimization technique, when at least one constraine is violed the objective function is set to a very small value:

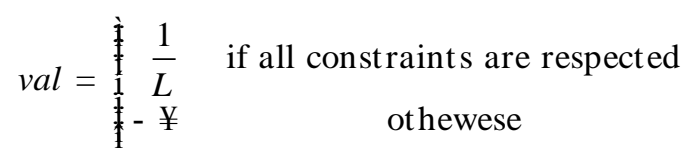

Where $L$ is a composite function of (8, 9 and 10).

2) codage:

The number of opened lines is equal $N_{\text {off }}=\stackrel{\triangleright}{\mathrm{a}}_{k=1}^{M} u_{k}-N+N_{\text {feeder }}$, where $N_{\text {feeder }}$ is the number of feeders. Hence, $N_{\text {off }}$ strings should be used. Each string is coded by a binary way, with a length equal to:

$$
\operatorname{fix}(\ln (M))+1
$$

Where fix is a round towards zero function.

\section{3) Initialization:}

The starting population is chosed randomly. However, to ensure the research in the feasible space (especialy the respect of the constraint (4)) the original situation is used.

4) Selection:

To maintaining the elite invidious having a great fitness function, the selection should be competitive. In this paper, the 'roulette wheel selection' scheme is used where each string lodges an area of the wheel that is equal to the string's share of the total fitness.

\section{5) Crossover}

The crossover operation is perform on random two invidious from the mating pool and produces two new invidious, each will result from one part of the parent string. Mutation gives a technique to generate new information into the knowledge base.

\section{6) Mutation}

A portion of the children individuals will have some of their bits flipped. Its purpose is to maintain diversity within the 
population and inhibit premature convergence. Mutation alone induces a random walk through the search.

\section{CASE STUDY}

The Distribution power system used is the 106 buses and 03 feeders LV (400 V) network of the R'mali Medea city (Figure $3)$.

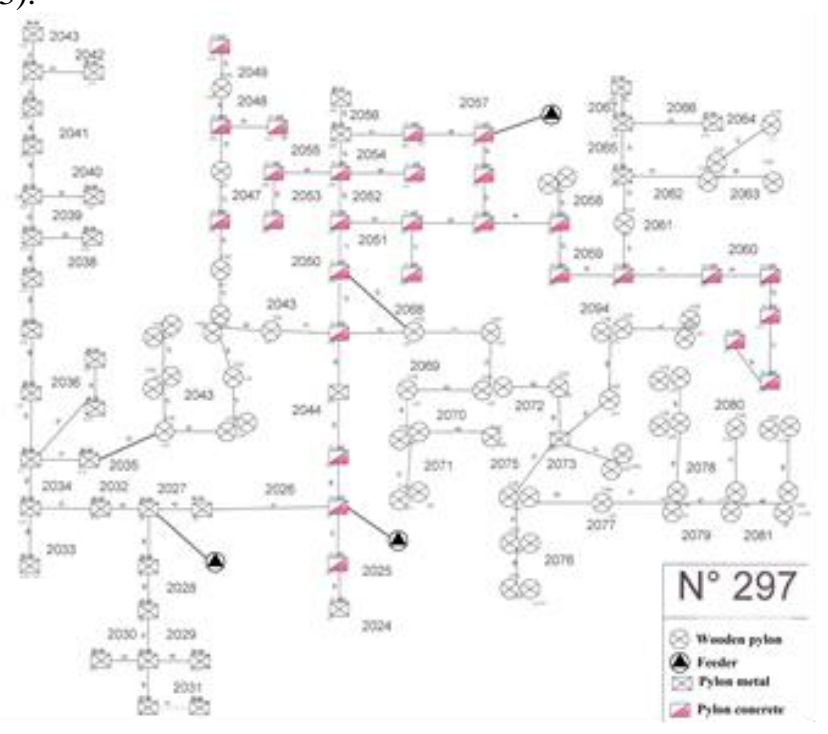

Fig. 3. R'Mali P297 Power system

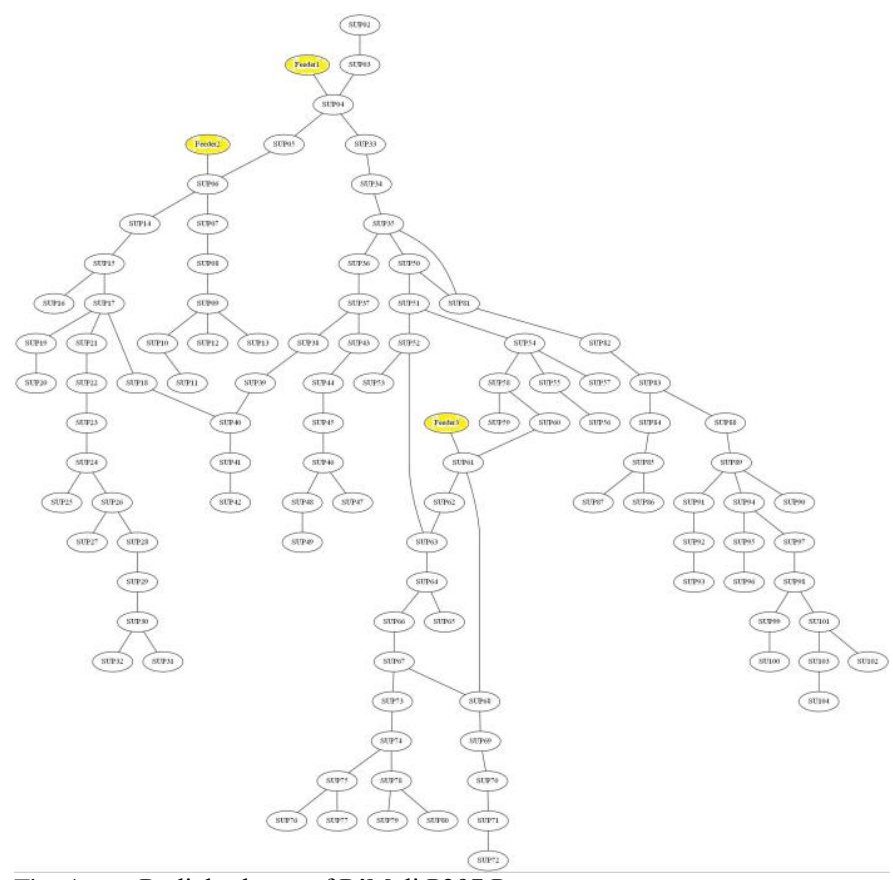

Fig. 4. Radial scheme of R'Mali P297 Power system

Figure 4 show that this network contain six dependents loops. Hence, six lines should be switched off. To find these lines, the problem presented in (1) should be resolved, it is clear that it is an Integer Non Linear Programing (INLP) problem. The parameters of the Genetic Algorithms (GA) are described in the Table I.
For the comparison, the classical method B\&B is used.

The table 1, conform the superiority of the GA then the B\&B.

TABLE I

GA PARAMETER

\begin{tabular}{|l|c|}
\hline \multicolumn{1}{|c|}{ Parameter } & Values \\
\hline Population size & 20 \\
\hline Maximum generation & 200 \\
\hline Crossover probability & 0.04 \\
\hline Mutation probability & 0.08 \\
\hline
\end{tabular}

TABLE II

COMPARISON GA AND B\&B FOR R'MALI DPS

\begin{tabular}{|c|c|c|c|c|}
\hline Method & $\begin{array}{c}\text { switched off } \\
\text { lines }\end{array}$ & Obj. (8) in $\mathrm{kW}$ & Obj. (9) & Obj. (10) \\
\hline $\begin{array}{c}\text { Genetic } \\
\text { Algorithms }\end{array}$ & $\begin{array}{l}\text { SUP35 SUP36 } \\
\text { SUP52 SUP63 } \\
\text { SUP04 SUP05 } \\
\text { SUP35 SUP50 } \\
\text { SUP50 SUP81 } \\
\text { SUP66 SUP67 }\end{array}$ & 2.7666 & 1.0765 & $5.23 \%$ \\
\hline $\begin{array}{c}\text { Branch and } \\
\text { Bound }\end{array}$ & $\begin{array}{l}\text { SUP51 SUP54 } \\
\text { SUP37 SUP38 } \\
\text { SUP04 SUP05 } \\
\text { SUP35 SUP50 } \\
\text { SUP50 SUP81 } \\
\text { SUP66 SUP67 }\end{array}$ & 2.7811 & 1.0765 & $5.66 \%$ \\
\hline
\end{tabular}

It is clear that the three objectives are not conflicting; the optimization of one from these three objectives lead to the optimization of the two others.

The Genetic Algorithms is very time consuming due to searching on the unfeasible region, the solution of this problem is dedicated to our next work.

\section{CONCLUSION}

This paper proposes the application of a met-heuristic optimization technique called Genetic Algorithms (binary coded) for the reconfiguration of the Distribution Power System to minimize the loses, improving the load-ability and the voltage profile. For the comparison the Branch and Bound technique, which is used by the Algerian company, is implemented. To conclude, the GA is clearly superior to B\&B. As the GA is very time consuming; in our future work; we will improve this weakness due to the search tower the unfeasible space.

\section{REFERENCES}

[1] Gonen T. Electric power distribution system engineering. 1st ed. New York: McGraw Hill; 1986.

[2] Sarfi RJ, Salama MMA, Chikhani AY. A survey of the state of the art in distribution system reconfiguration for system loss reduction. Electr Power Syst Res 1994;31(1):61-70.

[3] Kalantar M, Dashti R, Dashti R. Combination of network reconfiguration and capacitor placement for loss reduction in distribution system with based genetic algorithm. In: Proc 41st international universities power engineering conf Newcastle upon Tyne UK, vol. 1; 2006. p. 308-12.

[4] Khator SK, Leung LC. Power distribution planning: a review of models and issues. IEEE Trans Power Syst 1997;12(3):1151-9.

[5] Civanlar S, Grainger JJ, Yin H, Lee SSH. Distribution feeder reconfiguration for loss reduction. IEEE Trans Power Deliver 1988;3(3). p. 1227-3.[6] Baran ME, Wu FF. Network reconfiguration in distribution systems for loss reduction and load balancing. IEEE Trans Power Deliver 1989;4(2):1401-7. 
[6] Goswami SK, Basu SK. A new algorithm for the reconfiguration of distribution feeders for loss minimization. IEEE Trans Power Deliver 1992;7(3):1484-91.

[7] Gomes FV, Carneiro Jr S, Pereira JLR, Vinagre MP, Garcia PAN. A new heuristic reconfiguration algorithm for large distribution systems. IEEE Trans Power Syst. 2005;20(3):1373-8

[8] Gomes FV, Carneiro Jr. S, Pereira JLR, Vinagre MP, Garcia PAN, Oliveira EJ, Araújo LR. A new distribution system reconfiguration approach using optimal power flow technique and sensitivity analysis for loss reduction. In: IEEE Proc Power Engineering Society General Meeting San Francisco, CA, USA, vol. 1(1); 2005. p. 1-5

[9] Raju GKV, Bijwe PR. An efficient algorithm for loss reconfiguration distribution system based on sensitivity and heuristics. IEEE Trans Power Syst 2008;23(3):1280-7.

[10] Oliveira LW, Oliveira EJ, Carneiro Jr S, Pereira JLR, Costa JS, Silva J IC. Optimal reconfiguration and capacitor allocation in radial distribution systems energy losses minimization. Int $\mathrm{J}$ Electric Power Energy 2010;32(8):840-8.

[11] Nara K, Shiose A, Kitagawa M, Ishihara T. Implementation of genetic algorithm for distribution systems loss minimum re-configuration. IEEE Trans Syst 1992;7(3):1044-51.

[12] Torres-Jimenez J, Guardado JL, Rivas F, Maximov S, Melgoza E. Reconfiguration of power distribution systems using genetic algorithms and spanning trees Proc electronics, robotics and automotive mechanics conference Cuernavaca morelos Mexico; 210. p. 779-784.

[13] Torres J, Guardado JL, Rivas-Dávalos F, Maximov S, Melgoza E. A genetic algorithm based on the edge window decoder technique to optimize distribution systems reconfiguration. Int $\mathrm{J}$ Electric Power Energy 2013;45(1):28-34.

[14] Mirhoseini S H, Hosseini SM, Ghanbari M, Ahmadi M. A new improved adaptive imperialist competitive algorithm to solve the reconfiguration problem distribution systems for loss reduction and voltage profile improvement Electric Power Energy Syst 2014;55(1):128-43.

[15] Mazza A, Chicco G, Russo A. Optimal multi-objective distribution system reconfiguration with multi criteria decision making-based solution ranking and enhanced genetic operators. Int $\mathbf{J}$ Electric Power Energy 2014;54(1):255-67.

[16] Chiang H, Jean-Jumeau R. Optimal network reconfiguration in distribution system. Part 2: solution algorithms and numerical results. IEEE Trans Deliver 1990;5(3):1568-74.

[17] Zhigang M. Study on distribution network reconfiguration based on genetic simulated annealing algorithm. In: Proc China international conference electricity distribution guangzhou China; 2008. p. 1-7.

[18] Khoa TQD, Phan BTT. Ant colony search-based loss minimum reconfiguration of distribution systems. In: IEEE proc power india conference New Delhi, India; 2006. p. 6.

[19] Mori H, Ogita Y. A parallel tabu search based method for reconfigurations distribution systems. IEEE Proc Summer Power Eng Soc Meeting Seattle USA 2000;1:73-8.

[20] Mekhamer SF, Abdelaziz AY, Mohammed FM, Badr MAL. A new intelligent optimization technique for distribution systems reconfiguration. In: Proc international middle-east power system conference Aswan Egypt; 2008 397-401.

[21] Kumar KS, Jayabarathi T. Power system reconfiguration and loss minimization for an distribution systems using bacterial foraging optimization algorithm J Electric Power Energy Syst 2012;36(1):13-7.

[22] Hooshmanda R, Soltanib SH. Simultaneous optimization of phase balancing and reconfiguration in distribution networks using BF-NM algorithm Electric Power Energy Syst 2012;41(1):76-86.

[23] Boardman, J.T.; Meckiff, C.C.,"A Branch and Bound Formulation to an Electricity Distribution Planning Problem", IEEE, Transaction on PAS, Vol. PAS-104, no 8, Aug., 1985, pp. 2112-2117.

[24] Berge, C. "Graphes", Gauthiers-Villars, Paris, 1986.

[25] D. E. Goldberg "Genetic Algorithms in Search, Optimization, and Machine Learning”. Addison-Wesley Longman, USA, 1989. 